\title{
Holographic study of the QCD matter under external conditions
}

\author{
Alisa Katanaeva ${ }^{1, a}$ and Sergey Afonin ${ }^{1}$ \\ ${ }^{1}$ Department of High Energy and Elementary Particles Physics, Saint Petersburg State University, \\ 1 ul. Ulyanovskaya, 198504, Russia
}

\begin{abstract}
Holographic QCD is based on the AdS/CFT duality and offers new nonperturbative approaches to understand the strongly interacting regime of gauge theories. One of the primary questions in Quantum Chromodynamics is the clarification of the whole phase diagram of matter out of quarks and gluons as a function of temperature, baryon or quark chemical potential, and other external parameters.

We use methods of the bottom-up AdS/QCD approach to bring out the phase structure of several holographic models in which transition to a deconfined phase is related to a (first order) Hawking-Page phase transition. The impact of phenomenological model parameters on the critical temperature and chemical potential is studied in detail. Comparison of the model predictions with results of experimental investigations, lattice QCD simulations and other methods is also done.
\end{abstract}

\section{Introduction}

The established duality between a string theory on $A d S_{5} \times S_{5}$ and the supersymmetric Yang-Mills theory on the boundary $\partial A d S_{5}$, called the AdS/CFT correspondence, can be viewed as an example of a general correspondence between weakly coupled theories of gravity in 5 dimensions and strongly coupled quantum field theories in 4 dimensions. This hypothesis was naturally regarded as a way to find a holographical dual to QCD in the large $N_{c}$ limit and thus as a new tool to handle the nonperturbative regime of strong interactions. This holographic approach to QCD is giving insight into many QCD phenomena, including confinement, chiral symmetry breaking, structure of the phase diagram etc.

We focus on the bottom-up approach which relies on the consideration that a model in a gravity frame that provides a boundary theory incorporating the phenomenological features of real QCD is worth of the exploration. In other words, the goal is to reproduce the QCD phenomenology, but the model building follows the main prescriptions of AdS/CFT. Further we list essential methods and properties of the AdS/QCD correspondence:

- operators $O(x)$ in the $4 D$ theory are dual to fields $\phi(x, z)$ in the $5 D$ dual theory;

- a canonical dimension $\Delta$ of a $p$-form operator $O(x)$ is responsible for the $5 D$ mass of $\phi(x, z)$, which is given by the equation $m_{5}^{2}=(\Delta-p)(\Delta+p-4)$;

- global symmetries in the $4 D$ theory correspond to gauge symmetries on the $5 D$ side;

\footnotetext{
ae-mail: alice.katanaeva@gmail.com
} 
- a source $\phi_{O}(x)$ of an operator corresponds to the value of the $5 D$ field on the boundary $\phi(x, \epsilon)$;

- the generating functional of the connected correlators in the 4D theory and the effective action of the 5D theory are equivalent: $W_{4 D}\left[\phi_{O}(x)\right]=S_{5 D, e f f}[\phi(x, \epsilon)]$ with $\phi(x, \epsilon)=\phi_{O}(x)$;

- differentiating $S_{5 D, e f f}$ with respect to $\phi_{O}$ brings the QCD Green's functions;

- the deconfinement phase transition can be regarded as being dual to a Hawking-Page phase transition at some critical values of the parameters between a low temperature thermal AdS space (the confining phase) and a high temperature black hole (the deconfined phase) in AdS/QCD models.

As an example of the first three points consider the $S U\left(N_{f}\right)$ vector current $\bar{q} \gamma^{\mu} t^{a} q$ with $p=1$ and $\Delta=3$; it corresponds to the gauge field $A_{\mu}^{a}(x, z)$ with $m_{5}^{2}=0$.

The Regge behaviour of meson spectra is supposed to be a manifestaion of confinement in QCD and this property is a simple consequence of the Soft Wall (SW) hologrpahic model [1]. The model is given by a $5 \mathrm{D}$ action:

$$
S=-\frac{1}{4 g_{5}^{2}} \int d^{5} x \exp \left(-a z^{2}\right) \sqrt{-g} g^{M K} g^{L N} F_{M N} F_{K L},
$$

where $g=\operatorname{det} g_{M N}, F_{M N}=\partial_{M} V_{N}-\partial_{N} V_{M}, M, N,=0,1,2,3,4$ and the $A d S_{5}$ metric is parametrized as follows (with $L$ being the $A d S_{5}$ radius and $z$ - the extra fifth coordinate):

$$
g_{M N} d x^{M} d x^{N}=\frac{L^{2}}{z^{2}}\left(\eta_{\mu \nu} d x^{\mu} d x^{\nu}-d z^{2}\right) .
$$

Due to the quadratic form of the exponential factor in the dilaton term $e^{-\Phi(z)}$ the SW model provides linear radial Regge spectra:

$$
M_{n}^{2}=4 a(n+1)
$$

While even the result of Eqn. (3) can be considered as a phenomenological success, one can go further and try to achieve a more realistic spectrum with an arbitrary intercept:

$$
M_{n}^{2}=4 a(n+1+b) .
$$

The self-consistent way to introduce an arbitrary intercept consists in building the Generalized Soft Wall (GSW) model [2] for vector mesons:

$$
S=-\frac{1}{4 g_{5}^{2}} \int d^{5} x \exp \left(-a z^{2}\right) U^{2}\left(b, 0 ; a z^{2}\right) \sqrt{-g} g^{M K} g^{L N} F_{M N} F_{K L}
$$

where $U(\alpha, \beta ; x)$ denotes the Tricomi hypergeometric function $(U(0,0 ; x)=1)$. The GSW model preserves the ultraviolet and infrared asymptotes of the original SW model and gives the spectrum of Eqn. (4).

\section{The deconfinement temperature from AdS/QCD models}

To study the finite temperature effects assume that the thermodynamics is governed by the gravitational part of the action, which scales as $N_{c}^{2}$ (while the mesonic part $\sim N_{c}$ ). Further we follow the logic of the method for calculating the critical temperature $T_{c}$ proposed by Herzog [3].

In the SW model the gravitational part of the $5 \mathrm{D}$ action is:

$$
S_{S W}=\frac{1}{2 \kappa^{2}} \int d^{4} x d z e^{-\Phi(z)} \sqrt{-g}(\mathcal{R}-2 \Lambda),
$$


Table 1. Some predictions of $T_{c}$ based on the PDG data on vector mesons [5].

\begin{tabular}{|c|c|c|c|}
\hline Particle & Radial states & $m_{n}^{2}, \mathrm{GeV}^{2}$ & $T_{c}, \mathrm{MeV}$ \\
\hline$\rho$ & $n=0,1,2$ & $1.18(n+0.61)$ & 143 \\
$\omega$ & $n=0,1,2$ & $1.09(n+0.66)$ & 149 \\
$\rho$ & $n=0,1,2,3,4$ & $0.99(n+0.89)$ & 207 \\
$\omega$ & $n=0,1,2,3,4$ & $1.03(n+0.74)$ & 166 \\
$\rho$ & $n=0,1,2,4,5$ & $0.88(n+1.12)$ & 270 \\
$\omega$ & $n=1,2,3,4$ & $0.95(n+1.04)$ & 255 \\
- & "universal" slope [6] & $1.14(n+1)$ & 263 \\
\hline
\end{tabular}

where the dilaton profile $\Phi(z)=a z^{2}$ and $\Phi(z)$ is assumed not to affect the gravitational dynamics of the theory; $\mathcal{R}$ is the scalar curvature and $\Lambda$ - the cosmological constant.

On-shell the gravitational action (6) is the same for:

1) the thermal AdS metric: $d s^{2}=\frac{L^{2}}{z^{2}}\left(d t^{2}-d \vec{x}^{2}-d z^{2}\right)$, with $t \in[0, \beta]$ and $z \in[0, \infty]$;

2) the metric of AdS with a black hole: $d s^{2}=\frac{L^{2}}{z^{2}}\left(f(z) d t^{2}-d \vec{x}^{2}-\frac{d z^{2}}{f(z)}\right)$, where $f(z)=1-\left(z / z_{h}\right)^{4}$, $z_{h}$ being the horzon position; $t \in\left[0, \pi z_{h}\right]$ and $z \in\left[0, z_{h}\right]$.

The Hawking temperature, later identified with the temperature of deconfinement, is related to the black hole horizon $z_{h}$ via the relation $T_{c}=1 /\left(\pi z_{h}\right)$.

The free action densities $V$ identified with the regularized action $\left.S_{S W}\right|_{\text {on-shell }}$ are:

$$
V_{\mathrm{Th}}(\epsilon)=\frac{4 L^{3}}{\kappa^{2}} \int_{0}^{\beta} d t \int_{\epsilon}^{\infty} e^{-\Phi(z)} z^{-5} d z, V_{\mathrm{BH}}(\epsilon)=\frac{4 L^{3}}{\kappa^{2}} \int_{0}^{\pi z h} d t \int_{\epsilon}^{z_{h}} e^{-\Phi(z)} z^{-5} d z .
$$

The two geometries are compared at a radius $z=\epsilon$ where the periodicity in the time direction is locally the same, thus giving $\beta=\pi z_{h} \sqrt{f(\epsilon)}$.

The order parameter of the phase transition is defined by $\Delta V$ :

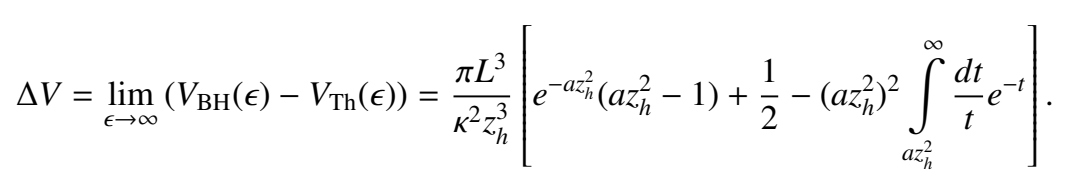

The procedure for the GSW model is the same and the resulting expression for $\Delta V$ involves the Tricomi functions carrying the intercept parameter $b$. Generally the Hawking-Page phase transition occurs at a point where $\Delta V=0$. Solving this equation with respect to $z_{h}$ gives the $T_{c}$ dependence on the slope parameter $a$ and the intercept parameter $b$.

The analysis of the impact of different meson trajectories parameters on the value of the deconfinement temperature was done in the paper [4]. We provide a Table 1 summarizing the major results.

For better understanding of the AdS/QCD predictions let us scope some of the lattice results. In presence of the physical quarks the deconfinement occurs as a cross-over in a range of temepratures, so to define the precise critical temperature can be an ambiguous task. Nevertheless, the modern methods [7] claim that $T_{c}$ lies in an interval of $150-170 \mathrm{MeV}$. That seems quite a successful prediction concerning that experimental results favour the range of $150-160 \mathrm{MeV}$ [8].

One can argue, however, that according to the philosophy of the AdS/QCD correspondence, the gravitational part of the holographic action (6) is dual to pure gluodynamics in the large- $N_{c}$ limit. Hence, the predicted value for $T_{c}$ must be compared with the lattice results for gluodynamics (i.e. with non-dynamical quarks) extrapolated to large $N_{c}$. Such extrapolations are given in [9]. The result is given as the first two terms of $1 / N_{c}^{2}$ expansion: $T_{c} / \sqrt{\sigma}=0.5949(17)+0.458(18) / N_{c}^{2}$. Fixing the 
value $\sqrt{\sigma}=420 \mathrm{MeV}$ as it is usually considred, we can get either $T_{c}=250 \mathrm{MeV}$ for $N_{c}=\infty$, or we can artificially put large $N_{c}=3$ and get $T_{c}=271 \mathrm{MeV}$. Furthermore, this point of view on the duality is more self-consitent. The general difficulty of identifying the deconfinement phase transition with the Hawking-Page one is that the last is of the first order. This problem is not present if we consider the 5D model being dual to the pure Yang-Mills $S U(N), N \rightarrow \infty$ theory as it suffers the same first order phase transition as soon as $N \geq 3$.

We considered three hypotheses in Table 1 . In the first one, only the well-established states are used. In this case, the predicted value of $T_{c}$ lies a bit below the interval given by lattices with dynamical light quarks and the $\rho$ and $\omega$ sectors yield close results. Next we add the poorly known states: the $\rho$ and $\omega$ sectors result in quite different predictions. Most likely, this is related to an insufficient accuracy of the experimental data. The last hypothesis consists in taking into account the states leading to a prediction of $T_{c}$ in the interval $250-270 \mathrm{MeV}$ expected from gluodynamics. Fascinatingly, following the idea of existence of the "universal" slope [6] we also get a prediction in this range. Considering the aforementioned points about the $S U(N)$ theory we regard this result as a check of our expectations: the universality of radial meson trajectories rising from the universal gauge behaviour should provide predictions in accordance with lattice simulations for gluodynamics.

\section{The temeprature - quark chemical potential phase diagram}

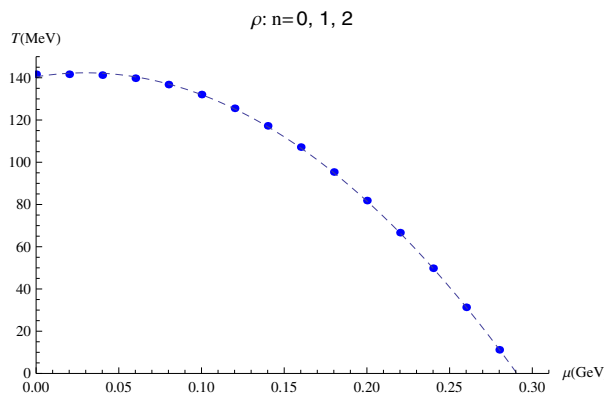

$\rho$ meson, $n=0,1,2-\left(T_{c}=143 \mathrm{MeV}, \mu_{B}=0\right)$ and $\left(T=0, \mu_{B} \simeq 0.9 \mathrm{GeV}\right)$.

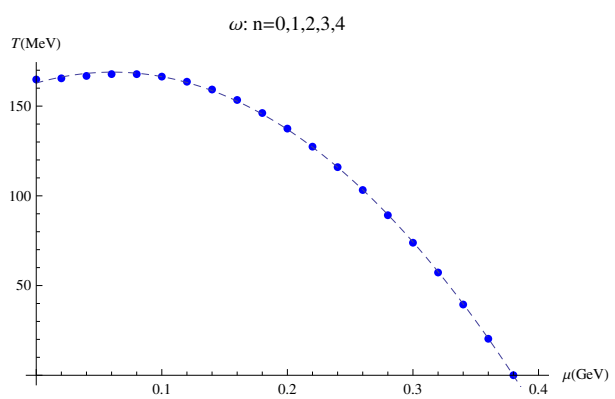

$\omega$ meson, $n=0,1,2,3,4-\left(T_{c}=166 \mathrm{MeV}\right.$, $\left.\mu_{B}=0\right)$ and $\left(T=0, \mu_{B} \simeq 1.1 \mathrm{GeV}\right)$.

Figure 1. Phase diagrams (see Eqn. (10)) on the $\left(T, \mu_{q}\right)$ plane with their endpoints for two particular trajectories.

In QCD the quark chemical potential $\mu_{q}$ is introduced coupled to the quark number current $\mu_{q} \bar{\psi} \gamma_{0} \psi$. Following the AdS/CFT dictionary we should introduce a dual 5D $U(1)$ field in a bulk of $A d S_{5}$ for this vector current. Consider the GSW Euclidean action consisting of the gravitational part and the $U(1)$ kinetic term:

$$
S=\int d^{4} x d z \sqrt{g} e^{-a z^{2}} U^{2}\left(b, 0 ; a z^{2}\right)\left(-\frac{1}{2 \kappa^{2}}\left(\mathcal{R}+12 / L^{2}\right)+\frac{1}{4 g_{5}^{2}} F_{M N} F^{M N}\right)
$$

As expected we get the solution of the Maxwell equations leaving non-zero only the time component of the vector field: $A_{i}=A_{z}=0, A_{t}=i\left(\mu-Q z^{2}\right)$. At small $z$ the dominant first term $\mu$ is identified with the source of the operator on the boundary, i.e. with the value of quark chemical potential, while the second term gives the expectation value of the operator, i.e. $Q$ should be referred to as quark number density. 
Similar to the construction of Section 2 and following [10] we get two geometries, both being solutions of the Einstein equations but corresponding to different phases:

1) the thermal charged AdS corresponding to the confining phase with the metric

$d s_{t c}^{2}=\frac{L^{2}}{z^{2}}\left(f_{t c}(z) d t^{2}+d \vec{x}^{2}+\frac{1}{f_{t c}(z)} d z^{2}\right)$, where $f_{t c}(z)=1+q^{\prime 2} z^{6}$

2) the Reissner-Nordstrem black hole in AdS corresponding to the deconfined phase with the metric $d s_{R N}^{2}=\frac{L^{2}}{z^{2}}\left(f_{R N}(z) d t^{2}+d \vec{x}^{2}+\frac{1}{f_{R N}(z)} d z^{2}\right)$, where $f_{R N}(z)=1-m^{2} z^{4}+q^{2} z^{6}$.

Naturally, the two geometries contain charges. The BH charge and quark number density are connected via $Q=\sqrt{\frac{3 g_{5}^{2} L^{2}}{2 \kappa^{2}}} q$. Both $q$ and $q^{\prime}$ can be related to the quark chemical potential $\mu$ (the details for the SW model could be seen in [10]).

The position of the phase transition can be found from the equation $\Delta \bar{S}=\bar{S}_{R N}-\bar{S}_{t c}=0$, where $\bar{S}$ implies a regularization by means of extracting the action (9) evaluated on the thermal AdS metric.

Setting the phenomenological parameters $a, b$ we determine the position of $z_{h}$ and get a curve on the $(T, \mu)$ plane defining the phase structure:

$$
T=-\left.\frac{1}{4 \pi} \frac{\partial f_{R N}}{\partial z}\right|_{z=z_{h}}=\frac{1}{\pi z_{h}}-\frac{1}{3 \pi} \frac{\kappa^{2}}{g_{5}^{2} L^{2}} \mu^{2} z_{h}
$$

For a numerical calculation of the phase diagram we must fix the combination $\frac{\kappa^{2}}{g_{5}^{2} L^{2}}$. We propose a novel way to fix this quantity which, to some extend, follows from QCD. The normalization of $g_{5}$ stems from the ultraviolet logarithmic asymptote of the quark vector current two-point correlation function $\left\langle J_{V} J_{V}\right\rangle$. Further, the pure gravitational part of the holographic action is regarded as dual to the large- $N_{c}$ pure gluodynamics in which the scalar sector determines the vacuum of planar QCD. Then, in the same spirit, one can construct the gluon scalar current of lowest dimension $J_{G}=\operatorname{Tr}\left(G_{\mu \nu}\right)^{2}$ and consider the correlator $\left\langle J_{G} J_{G}\right\rangle$. Its ultraviolet asymptote fixes the parameter $k$ and we have $\frac{\kappa^{2}}{g_{5}^{2} L^{2}}=\frac{1}{12 N_{c}}$. Eventually, the model predicts the phase diagrams like those in Figure 1. The region below the curve corresponds to the confining phase, over - to the deconfined, and the curve itself - to the first order phase transition.

Consider the endpoints where the critical value of the baryon chemical potential $\mu_{B c}$ is finite and $T_{c}=0$ in the diagrams from Figure 1 (there the $x$ axis is $\mu=\mu_{q}=1 / 3 \mu_{B}$ ). They match our expectations that $\mu_{B C}$ is about the nucleon mass and lie close to a range to which the experimental data converge: $\mu_{B c} \simeq 1.1 \div 1.2 \mathrm{GeV}[8]$.

\section{Isospectral models}

Consider an idea suggested in [11] and inspired by the SUSY quantum mechanics of constructing a family of strictly isospectral potentials associated with a given potential for Schrödinger-type equations. In the context of the AdS/QCD approach we can use this to modify the dilaton profile $\Phi(z)$ and have a tower of new models while keeping the spectrum fixed. The family members are distinguished through a parameter $\lambda$, with $\lambda=\infty$ corresponding to the original spectrum. For the SW model the dilaton profiles are the solutions of the following equation in terms of $t=a z^{2}$ :

$$
2 t \Phi^{\prime \prime}(t)-t \Phi^{\prime 2}(t)+t=4\left(\frac{d}{d t}+2 t \frac{d^{2}}{d t^{2}}\right) \ln \left(1+\lambda-\int_{t}^{\infty} t^{\prime} e^{-t^{\prime}} d t^{\prime}\right) .
$$

Further, the Eqn. (8), defining the position of the phase transition transforms into: $\Delta V=$ $\frac{\pi R^{3}}{k z_{h}^{3}}\left[e^{-\Phi\left(a z_{h}^{2}\right)}\left(-1+a z_{h}^{2} \Phi^{\prime}\left(a z_{h}^{2}\right)\right)+\frac{1}{2}+\left(a^{2} z_{h}^{4}\right) \int_{a z_{h}^{2}}^{\infty} d t\left(\Phi^{\prime \prime}(t)-\Phi^{\prime 2}(t)\right) t^{-1} e^{-\Phi(t)}\right]=0$. These modifications could af- 
fect greatly the predicted critical temperature. For instance, for the "universal" slope fit $T_{c}$ changes from $263 \mathrm{MeV}$ at $\lambda=\infty$ to $195 \mathrm{MeV}$ at $\lambda=1$ and $154 \mathrm{MeV}$ at $\lambda=0.01$. And for the $n=0,1,2,3,4$ $\rho$-mesons from the third row of Table $1 T_{c}$ changes from $206 \mathrm{MeV}$ at $\lambda=\infty$ to $161 \mathrm{MeV}$ at $\lambda=1$ and less for smaller values of $\lambda$. The general tendency is obvoius: as $\lambda$ decreases $T_{c}$ decreases also.

Another possibilty in this framework is to consider a 5D action for another type of bosonic modes - for the scalar fields. This model could be treated as a holographic description of the scalar glueballs. Interestingly, in contrast to the vector case, we'll get approximately the same $T_{c}$ for the whole isospectral family then. For example, take the lattice scalar glueball spectrum $M_{n}^{2}=4.5(n+0.56) \mathrm{GeV}^{2}[12]$, then the predicted $T_{c} \simeq 139 \div 140 \mathrm{MeV}$.

\section{Conclusions}

This work is devoted to the determination of properties of some AdS/QCD models on the $\left(T, \mu_{B}\right)$ plane within the bottom-up holographic approach. The possibilty to get predictions for medium and large values of $\mu_{B}$ is especially important, as another nonperturbative method - the calculations on lattice - fails in this region.

We have analysed SW and GSW model for the value of $T_{c}$. We wish to emphasize that from the point of view of the AdS/QCD ideology the predicted $T_{c}$ must refer to the deconfinement phase transition in the pure gluodynamics $(\sim 250 \mathrm{MeV})$. The best prediction comes from the most general "universal" slope fit. We looked into the finite $\mu_{B}$ behaviour in the GSW model and found the result depending on the input phenomenological parameters. Our position is that the curve of phase transition becomes ambiguous mostly because of lack of sufficient amount of reliable experimental data on the radially excited light mesons. The use of well established states gives the phase structure similar to the experimental and lattice (with dynamical quarks) results.

We have marked down our current field of interest: isospectral AdS/QCD models. They manifest that the SW models are not fixed by the form of the meson spectra only and therefore suggest something unexplored in the field. It remains an open question whether this new freedom would just help to better adjust to phenomenology or give other deeper insights.

\section{Acknowledgements}

The work was supported by Saint Petersburg State University and by the RFBR grant 16-02-00348-a.

\section{References}

[1] A. Karch, E. Katz, D. T. Son and M. A. Stephanov, Phys. Rev. D 74, 015005 (2006).

[2] S. S. Afonin, Phys. Lett. B 719, 399 (2013).

[3] C. P. Herzog, Phys. Rev. Lett. 98, 091601 (2007).

[4] S. S. Afonin, A.D.Katanaeva, Eur. Phys. J. C, 74, 3124 (2014).

[5] J. Beringer et al. (Particle Data Group), Phys. Rev. D 86, 010001 (2012).

[6] D. V. Bugg, Phys. Rept. 397, 257 (2004).

[7] S. Borsanyi et al. [Wuppertal-Budapest Collaboration], JHEP 1009, 073 (2010).

[8] A. Andronic et al., Nucl. Phys. A 837, 65 (2010)

[9] B. Lucini, A. Rago and E. Rinaldi, Phys. Lett. B 712, 279 (2012).

[10] C. Park, D. Gwak, B. Lee, Y. Ko, and S. Shin, Phys. Rev. D 84, 046007 (2011).

[11] A. Vega and P. Cabrera, Phys. Rev. D 93, 114026 (2016).

[12] H. B. Meyer, Glueball Regge trajectories, Ph.D. Thesis [hep-lat/0508002]. 